\title{
Caractérisation en biovars d'isolats marocains d'Agrobacterium issus de tumeurs racinaires des rosacées fruitières
}

\author{
A. Benjama ${ }^{1}$ et S. Daoud ${ }^{2}$ \\ 1 INRA, laboratoire de phytobactériologie, B.P. S/40, Meknès; \\ 2 Faculté des Sciences, Meknès, Maroc
}

(reçu le 7 juin 1988; accepté le 4 juillet 1989)

Résumé - Cette étude porte sur la caractérisation et le contrôle du pouvoir pathogène de 103 isolats d'Agrobacterium à partir d'une synthèse des tests différents proposés dans la littérature. Quarante-quatre souches d'Agrobacterium ont pu être alors identifiées dont 22 sont pathogènes. Les résultats montrent que les tumeurs à Crown-gall de l'amandier, du prunier et du pêcher de la région de Meknès, Maroc, contiennent des Agrobacterium pathogènes ou non pathogènes; le biovar 2 ( 26 souches dont 14 pathogènes) s'y trouve plus fréquemment que le biovar 1 (14 souches dont 8 pathogènes).

Une gamme de plantes indicatrices : tomate, tournesol, tabac permet de révéler une proportion d'agents pathogènes plus élevée que l'inoculation d'une seule espèce végétale.

crown-gall - amandier - prunier - pêcher - biovar

Summary - Biovar caracterization of moroccan Agrobacterium isolates originating from root tumors on deciduous fruit trees. Determination of the physiological characters of Agrobacterium and ways of testing for pathogenicity vary from author to author. It was therefore necessary for a combination of tests to be followed in this study. 44 strains of Agrobacterium were identified, 22 of which are pathogenic. It has been shown, among the pathogenic and non-pathogenic strains in tumors of almond, plum and peach, that biovar 2 (26 strains) occurs more frequently than biovar 1 (14 strains) in the Meknès area.

When used together to check pathogenicity test-plants like tomato, sunflower and tobacco revealed more pathogenic bacteria than a single test plant.

crown-gall - almond - plum - peach - biovar

\section{INTRODUCTION}

La galle du collet ou Crown-gall des rosacées fruitières sévit au Maroc. L'importance et la répartition de cette maladie a été déjà décrite (Benjama et al., 1987). La région de Meknès avec plus de $50 \%$ de la production arboricole nationale est la plus touchée. Les pépinières sont les principaux foyers de la maladie où le nombre de plants atteints de tumeurs provoquées par Agrobacterium peut atteindre $90 \%$.

La taxonomie d'Agrobacterium a fait l'objet de beaucoup de controverses. Différents auteurs utilisent des appellations différentes. Allen \& Holding (1974), mentionnent Agrobacterium tumefa- ciens qui est synonyme d'A. radiobacter d'après Keane et al. (1970), White (1972), Kersters et al. (1973), Holmes \& Roberts (1981). Ces derniers rejettent l'appellation $A$. radiobacter si $A$. tumefaciens est retenu comme espèce-type.

La nomenclature d'Agrobacterium était confuse, car basée sur le pouvoir pathogène qui est transférable de bactérie en bactérie (Kerr \& Brisbane, (1983). D'après ces mêmes auteurs, Agrobacterium provoque deux maladies : la galle du collet (Crown-gall) et la galle chevelue (Hairy-root). Les trois espèces d'Agrobacterium ( $A$. tumefaciens, $A$. rhizogenes, $A$. rubi) provoquent le Crown-gall si elles portent le plasmide $\mathrm{Ti}$ alors que seulement les deux premières espèces 
donneraient le Hairy-root si elles portent le plasmide Ri.

Ce qui frappe, dans l'analyse de Kerr \& Brisbane (1983) c'est qu'il n'est plus question de pathovar au sens de Kerr et al. (1978), ni de variété comme le préconisent Keane et al. (1970), New \& Kerr (1972), Kerr \& Panagopoulos (1977), Panagopoulos et al. (1978), mais de biovar d'après Kersters et De Ley (1984).

Ces différentes approches témoignent de la complexité du genre Agrobacterium et de son hétérogénéité. Les différents auteurs n'épousent pas souvent le même schéma d'identification ce qui laisse un doute sur la nomenclature à utiliser. Dans cette étude, il a été tenu compte de cette variabilité d'approche en adoptant les tests physiologiques communs utilisés par Keane et al. (1970), White (1972), Kerr (1974), Kerr \& Panagopoulos (1977), Dhanvantari (1978), Spiers (1979), Schaad (1980), Miller \& Vruggink (1981), Bazzi \& Rosciglione (1982), Knauf et al. (1982), Kersters \& De Ley (1984) (Tableau I). La caractérisation des isolats d'Agrobacterium en biovar est accompagnée du test de leur pouvoir pathogène sur plantes indicatrices et l'appellation de la souche pathogène sera conforme à celle proposée par Kersters \& De Ley (1984).

\section{MATÉRIELS ET MÉTHODES}

\section{Souches bactériennes}

L'origine des souches bactériennes et leurs hôtes respectifs figurent dans le Tableau II. Elles sont isolées de tumeurs jeunes portées par les racines d'arbres âgés de 6 à 8 ans.

Tableau I. Synthèse des caractères physiologiques utilisés pour l'identification des biovars d'Agrobacterium.

Caractère Biovar, Biovar $_{2}$ Biovar $_{3}$

$\begin{array}{lccc}\text { 3-Cétolactose } & + & - & - \\ \text { Citrate de } \mathrm{Na} & - & + & + \\ \mathrm{NaCl} 2 \% & + & - & + \\ \text { Oxydase } & + & \mathrm{V} & \mathrm{V} \\ \mathrm{T}^{\circ} \text { max. de croissance } & 37^{\circ} \mathrm{C} & 29^{\circ} \mathrm{C} & 32^{\circ} \mathrm{C} \\ \text { Erythritol } & - & + & - \\ \text { Malonate } & - & + & - \\ \text { L+Tartrate } & - & + & -\end{array}$

V : Variable; + : réaction positive; - : réaction négative.

\section{Tests physiologiques}

Tous les tests sont réalisés au laboratoire à $20^{\circ} \mathrm{C}$.

\section{Production du 3-cétolactose}

La méthode de Bernaerts \& De Ley (1963) permet de distinguer les souches du biovar ${ }_{1}$ d'Agrobacterium qui sont positives de celles du biovar ${ }_{2}$ qui sont négatives.

Utilisation du citrate de sodium sur milieu de Simmons (1926).

\section{Tolérance au chlorure de sodium}

Le milieu de base est l'Agar nutritif (Nutrient broth $8 \mathrm{~g}$, gélose $15 \mathrm{~g}$ par litre d'eau distillée) auquel on ajoute $\mathrm{NaCl} 2 \%$. La croissance éventuelle est observée jusqu'à 5 j d'incubation.

\section{Recherche de l'oxydase par la méthode de Kovacs (1956).}

\section{Température maximale de croissance}

Les ensemencements bactériens sont placés à l'étuve à $27,29,37,39^{\circ} \mathrm{C}$ pendant $10 \mathrm{j}$.

\section{Utilisation différentielle des sources de carbone}

Le milieu d'Ayers et al. (1919) est utilisé comme milieu de base auquel on ajoute après autoclavage une solution stérilisée par filtration d'érythritol, glucose, saccharose, malonate ou $L(+)$ tartrate pour obtenir une concentration finale de $0,1 \%$.

L'observation de la croissance bactérienne se fait chaque j pendant une semaine.

\section{Pouvoir pathogène sur plantes indicatrices}

Des tranches de carotte sont inoculées par dépôt d'une suspension dense ( $10^{8}$ bactéries $/ \mathrm{ml}$ ) sur l'anneau vasculaire, puis maintenues en chambre humide à la température de $25^{\circ} \mathrm{C}$ (Ark \& Schroth, 1958). On note le développement des excroissances après $14 \mathrm{j}$.

Des jeunes plantes de tournesol, tomate et tabac sont utilisées au stade 3-4 feuilles. Des gouttes (1/20 $\mathrm{ml}$ ) de suspension bactérienne de $10^{8}$ bactéries $/ \mathrm{ml}$ sont déposées à 4 niveaux différents sur la tige blessée préalablement à l'aide d'une aiguille, à raison de 6 plantes par souche. Les jeunes plantes sont placées en chambre climatisée à $25^{\circ} \mathrm{C}$ et $70 \%$ d'humidité relative. Les résultats apparaissent au bout de $10 \mathrm{j}$ pour le tournesol, $15 \mathrm{j}$ pour la tomate et $30 \mathrm{j}$ pour le tabac. Si la bactérie est pathogène, une tumeur se développe au niveau de la blessure.

\section{Inoculation de Kalanchoe en vue de l'analyse des opines}

Quelques souches d'Agrobacterium pathogènes du biovar $_{2}$ sont inoculées sur Kalanchoe tubiflora de la même manière que sur tomate, tournesol et tabac. Une fois les tumeurs bien développées, elles sont envoyées à l'institut de microbiologie d'Orsay en vue de l'analyse des opines. 
Tableau II. Origine des souches d'Agrobacterium.

\begin{tabular}{|c|c|c|c|c|c|}
\hline Plante hôte & $N^{\circ}$ Tumeur & $N^{\circ}$ Souche & Pays & Date & Fournie par \\
\hline $\begin{array}{l}\text { Prunus amygdalus } \\
\text { (amandier amer) }\end{array}$ & 121 & $\begin{array}{l}\text { AT } 68 \\
121.7-121.9-121.19- \\
121.20-121.22-121.23- \\
121.27-121.35-121.39- \\
121.62-121.66-121.68- \\
121.71-121.84-121.85- \\
121.86-121.87-121.90\end{array}$ & $\begin{array}{l}\text { Grèce } \\
\text { Maroc } \\
\text { (Azrou) }\end{array}$ & $\begin{array}{r}1967 \\
1986\end{array}$ & Garrett \\
\hline & 136 & 136.4 & $\begin{array}{c}\text { Maroc } \\
\text { (Meknès) }\end{array}$ & 1986 & \\
\hline $\begin{array}{l}\text { Prunus domestica } \\
\text { (myrobolan) }\end{array}$ & $\begin{array}{r}72 \\
102 \\
103\end{array}$ & $\begin{array}{l}72.18 \\
102.9 \\
103.2-103.3-103.16- \\
103.18-103.21-103.30- \\
103.31-103.36\end{array}$ & $\begin{array}{c}\text { Maroc } \\
\text { (Meknès) } \\
\text { Maroc } \\
\text { (Meknès) } \\
\text { Maroc } \\
\text { (Meknès) }\end{array}$ & $\begin{array}{l}1984 \\
1985 \\
1985\end{array}$ & \\
\hline & $\begin{array}{l}134 \\
135\end{array}$ & $\begin{array}{l}134.2-134.10-134.13 \\
135.5\end{array}$ & $\begin{array}{l}\text { Maroc } \\
\text { (Meknès) } \\
\text { Maroc } \\
\text { (Meknès) }\end{array}$ & $\begin{array}{l}1986 \\
1986\end{array}$ & \\
\hline $\begin{array}{l}\text { Prunus persicae } \\
\text { (missour) }\end{array}$ & $\begin{array}{l}141 \\
142\end{array}$ & $\begin{array}{l}141.1-141.5-141.10- \\
141.11-141.12-141.13^{*} \\
142.5-142.7-142.8\end{array}$ & $\begin{array}{l}\text { Maroc } \\
\text { (Azrou) } \\
\text { Maroc } \\
\text { (Azrou) }\end{array}$ & $\begin{array}{l}1987 \\
1987 \\
1987\end{array}$ & \\
\hline
\end{tabular}

(*) $141.13: 2746$ CFBP Collection française de bactéries phytopathogènes, INRA, Angers, France.

\section{RÉSULTATS}

\section{Caractérisation des isolats d'Agrobacterium en biovar}

Parmi 103 isolats étudiés, seules 44 souches répondent aux caractéristiques d'Agrobacterium: aspect des colonies à l'isolement, Gram-, non fluorescent, oxydatif. Elles sont classées en 3 biovars (Tableau III) : 15 souches (dont 9 pathogènes) en biovar, 26 souches (dont 14 pathogènes) en biovar ${ }_{2}$; un biovar intermédiaire, répondant aux caractères des 2 biovars, a été appelé biovar $_{1-2}$; il comprend 3 souches non pathogènes.

\section{Test du pouvoir pathogène des isolats d'Agrobacterium}

Sur 44 souches bactériennes, seules 14 donnent des tumeurs sur tranches de carotte.
Sur tabac, 10 souches sont pathogènes, 17 sur tournesol et 21 sur tomate. Seulement 7 souches répondent positivement sur les 3 plantes (Tableau IV).

\section{Analyse des tumeurs sur Kalanchoe}

Les souches inoculées sur Kalanchoe tubiflora ont donné des tumeurs caractéristiques soit avec des petites racines sur toutes les tumeurs soit des bourgeons (Tableau V). Ces tumeurs analysées sont à Nopaline.

\section{DISCUSSION}

La caractérisation physiologique des Agrobacterium montre que la population bactérienne marocaine est variée et hétérogène. Le biovar ${ }_{2}$ est dominant par rapport au biovar, ${ }_{1}$ comme c'est le cas en Australie (New \& Kerr, 1972); en Grèce (Panagopoulos \& Psallidas, 1973); aux Etats- 
Tableau III. Classification en biovar des 44 souches d'Agrobacterium.

Biovar

$N^{\circ}$ de souche d'Agrobacterium

Total

Pathogène 1

Non pathogène

Pathogène 2

Non pathogène

Non pathogène 1-2
At $68-72.18-103.30-103.31-$ $103.36-121.62-121.68-141.1$. 141.5

$103.2-121.19-121.27-136.4-$

$141.10-142.5$

$102.9-103.16-103.18-103.21-$

$121.1-121.85-121.90-134.13-$

$135.5-141.11-141.12-141.13-$

$142.7-142.8$

$103.3-121.7-121.9-121.35-$

$121.39-121.66-121.71-121.84-$

$121.86-121.87-134.2-134.10$

$12(27 \%)$

$121.20-121.22-121.23$

Tableau IV. Réactions comparées de tomate, tournesol et tabac aux différentes souches d'Agrobacterium.

\begin{tabular}{|c|c|c|c|c|c|}
\hline & \multirow[t]{2}{*}{ Souches bactériennes } & \multicolumn{4}{|c|}{ Pathogénicité sur } \\
\hline & & tomate & tournesol & tabac & total \\
\hline \multirow{4}{*}{$\begin{array}{l}\text { Agrobacterium } \\
\text { Pathogène }\end{array}$} & $\begin{array}{l}\text { At } 68^{\star}-72.18-103.21^{\star}-134.13^{\star}- \\
135.5^{\star}-141.12^{\star \star}-142.8^{\star}\end{array}$ & + & + & + & 7 \\
\hline & $102.9-103.18^{\star}-103.36-121.62^{\star}-$ & & & & \\
\hline & $141.1-141.11-141.13^{\star}-142.7^{\star}$ & + & + & - & 8 \\
\hline & $103.30-121.1^{\star}-121.85^{\star}$ & + & - & + & 3 \\
\hline \multirow[b]{3}{*}{$\begin{array}{l}\text { Agrobacterium } \\
\text { non pathogène }\end{array}$} & $121.68^{\star}-121.90-141.5$ & + & - & - & 3 \\
\hline & $130.16-103.31$ & - & + & - & 2 \\
\hline & $\begin{array}{l}103.2-103.3-121.7-121.9-121.19- \\
121.20-121.22-121.23-121.27- \\
121.35-121.39-121.66-121.71 \\
121.84-121.86-121.87-134.2- \\
134.10-136.4-141.10-142.5\end{array}$ & - & - & - & 21 \\
\hline
\end{tabular}

+ : Présence de tumeur; - : Pas de tumeur; " : Souches ayant donné des tumeurs sur tranches de carotte.

Unis (Anderson \& Moore, 1979) et en France (Lopez, 1978). Cette hétérogénéité semble indépendante de l'origine de la souche puisqu'on trouve les biovars indifféremment sur prunier, amandier ou pêcher.

Le test du pouvoir pathogène des souches d'Agrobacterium nécessite plus d'une espèce végétale indicatrice pour vérifier le pouvoir tumorigène. La tomate et le tournesol donnent des réponses positives pour toutes les souches pathogènes testées (Tableau IV). La carotte et le tabac donnent des résultats restrictifs. II apparaît donc que le test sur carotte en boîte de Petri ou l'inoculation de plants de tabac ne sont pas 
Tableau V. Inoculation des Kalanchoe tubiflora et analyse des opines.

\begin{tabular}{|c|c|c|c|c|}
\hline Plante hôte & Biovar & $N^{\circ}$ souche & $\begin{array}{l}\text { Résultats sur } \\
\text { Kalanchoe* }\end{array}$ & $\begin{array}{c}\text { Analyse des } \\
\text { Opines }\end{array}$ \\
\hline \multirow[t]{3}{*}{ P. domestica } & 2 & 102.9 & $+\mathrm{BR}$ & $\mathrm{N}$ \\
\hline & 2 & 103.16 & + & \\
\hline & 2 & 103.21 & $+B R$ & $\mathrm{~N}$ \\
\hline \multirow[t]{4}{*}{ P. amygdalus } & 2 & 121.1 & $+B R$ & $N$ \\
\hline & - & 121.68 & & \\
\hline & 2 & 121.85 & $+B R$ & $N$ \\
\hline & 2 & 121.90 & $+\mathrm{BR}$ & $N$ \\
\hline \multirow[t]{3}{*}{ P. domestica } & 2 & 134.13 & $+R$ & $N$ \\
\hline & 2 & 135.5 & $+R$ & $N$ \\
\hline & & 141.5 & + & \\
\hline \multirow[t]{4}{*}{ P. persica } & 2 & 141.11 & $+R$ & $N$ \\
\hline & 2 & 141.12 & $+R$ & $N$ \\
\hline & 2 & 147.7 & $+R$ & $\mathrm{~N}$ \\
\hline & 2 & 142.8 & $+\mathrm{R}$ & $N$ \\
\hline
\end{tabular}

* - Test réalisé sur Kalanchoe tubiflora selon Paulus et al., 1989.

$\mathrm{N}$ - Tumeur analysée à Nopaline; B - Tumeur avec bourgeons; $\mathrm{R}$ - Tumeur avec racines.

"* - Analyse réalisée par le : Groupe de recherche interactions micro-organismes-plantes (Annick Petit). Université Paris XI. Institut de microbiologie.

91 405-Orsay.

fiables. Dans notre expérience, c'est le test d'inoculation sur tomate qui donne la réponse la plus large (21 souches positives), puis le tournesol (17 souches positives). L'utilisation couplée de ces 2 plantes-test permet une plus grande sécurité pour la détection des souches pathogènes.

Enfin notons qu'on peut trouver l'agent pathogène Agrobacterium (biovar ${ }_{1}$ et biovar $_{2}$ ) et un Agrobacterium non pathogène au niveau de la même tumeur : par exemple les souches 121 (Tableaux II, III, IV). Ceci montre la variabilité des souches au niveau du même site d'infection comme précédemment observé par Anderson \& Moore (1979) et Nesme et al. (1987).

Les agents pathogènes Agrobacterium sont des souches à Nopaline et sont donc sensibles à la K 84. Ceci nous permet d'envisager éventuellement la lutte biologique dans notre pays.

Dans des travaux futurs, l'accent sera mis sur l'étude sérologique des souches d'Agrobacterium en vue de leur détection en pépinière au Maroc, sur l'étude de la sensibilité variétale des différentes espèces fruitières vis-à-vis de l'agent pathogène et sur l'évaluation des possibilités de la lutte biologique et chimique.

\section{RÉFÉRENCES}

Allen N. \& Holding A.J. (1974) Genus II. Agrobacterium Conn. 1942. In : Bergey's manual of determinati- ve bacteriology, $8^{*}$ Edition. (R.E. Buchanan \& N.E. Gibbons eds) Williams \& Wilkins, Baltimore, 246-267

Anderson A.R. \& Moore L.W. (1979) Host specificity in the genus Agrobacterium. Phytopathology 69, 320-323

Ark A. \& Schroth M.N. (1958) Use of slices of carrot and other fleshy roots to detect crown-gall in soil. Plant Dis. Rep. 42, 1 279-1 281

Ayers S.H., Rupp P. \& Johnson W.T. (1919) Study on the alkali forming bacteria in milk. U.S. Depart. Agr. Bull. $782 \mathrm{P}$

Bazzi L. \& Rosciglione B. (1982) Agrobacterium

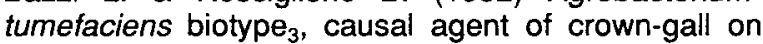
Chrysanthemum in Italy. Phytopathol. Z. 103, 280-284

Benjama A., Soussi M. \& Benyouness C. (1987) Studies on the crown-gall bacterium in Morocco. Arab and Near East Plant Prot. Newsl. 4, 16

Bernaerts M.J. \& De Ley J. (1963) A biochemical test for crown-gall bacteria. Nature, 197, 406-407

Dhanvantari B.N. (1978) Characterization of Agrobacterium isolates from stone fruits in Ontario. Can. J. Bot. 56, 2 309-2 311

Holmes B. \& Roberts P. (1981) The classification, identification and nomenclature of Agrobacteria incorporating revised descriptions for each of Agrobacterium tumefaciens (Smith and Townsend) Conn. 1942, Agrobacterium rhizogenes (Riker et al.) Conn. 1942 and Agrobacterium rubi (Hildebrant) Starr and Weiss 1943. J. Appl. Bacteriol. 50, 433-468

Keane P.J. Kerr A. \& New P.B. (1970) Crown-gall of stone fruit II. Identification and nomenclature of Agrobacterium isolates. Aust. J. Biol. Sci. 23, 585-595

Kerr A. (1974) Soil microbiological studies on Agrobacterium radiobacter and biological control of crown-gall. Soil Sci. 118-168 
Kerr A. \& Brisbane P.G. (1983) Agrobacteriun. In : Plant bacterial Diseases : a diagnostic guide (Fahy \& Persley, eds) Academic press, 27-43

Kerr A. \& Panagopoulos C.G. (1977) Biotypes of Agrobacterium radiobacter var. tumefaciens and their biological control. Phytopathol. Z. 90, 197-199

Kerr A., Young J.M. \& Panagopoulos C.G. (1978) A proposed nomenclature for plant pathogenic bacteria Genus II. Agrobacterium Conn. 1942. N. Z. J. Agr. Res., 21, 153-177

Kersters K. \& De Ley J. (1984) Genus Agrobacterium Conn. 1942. In : Bergey's Manual of Systematic Bacteriology (N.R. Krieg and J.C. Holt eds) vol. 1, 244254

Kersters K., De Ley J., Sneath P.H. \& Sackin M. (1973) Numerical taxonomic analysis of Agrobacterium. J. Gen. Microbiol. 78, 227-239

Knauf V.C., Panagopoulos C.G. \& Nester E.M. (1982) Genetic factors controlling the host range of Agrobacterium tumefaciens. Phytopathology, 72, 1545-1549

Kovacs N. (1956) Identification of Pseudomonas pyocyanea by oxidase reaction. Nature 178, 703

Lopez M. (1978) Characteristics of French isolates of Agrobacterium. Proc. 4 th. Int. Conf. Plant Pathogenic Bact., Angers 1, 233-238

Miller H.J. \& Vruggink J. (1981) An assessment of biochemical and serological tests for Agrobacterium radiobacter subsp. tumefaciens. Phytopathol. Z. 102300

Nesme X., Michel M.F. \& Digat B. (1987) Population heterogeneity of Agrobacterium tumefaciens in galls of
Populus L. from a single nursery. Appl. Environ. Microbiol. 53, 655-659

New P.B. \& Kerr A. (1972) A selective medium for Agrobacterium radiobacter biotype 2. J. Appl. Bacteriol. 34, 233-236

Panagopoulos C.G. \& Psallidas P.G. (1973) Characteristics of Greek isolates of Agrobacterium tumefaciens (Smith \& Townsend). J. Appl. Bacteriol. 30, 233-240

Panagopoulos C.G., Psallidas P.G. \& Alivizatos A.J. (1978) Studies on biotype 3 of Agrobacterium radiobacter var. tumefaciens. Proc. 4th. Int. Conf. Plant Pathogenic Bact. Angers 1, 221-228

Paulus F., Huss B., Bonnard G., Ridé M., Szegedi E., Tempé J., Petit A. \& Otten L. (1989) Molecular systematics of Biotype III $\mathrm{Ti}$ plasmids of Agrobacterium tumefaciens. Mol. Plant Microb. Inter. 2, 64-74

Schaad V.M. (1980) Gram negative bacteria. In : Laboratory Guide for Identification of Plant Pathogenic Bacteria. Amer. Phytopat. Soc. St. Paul, Minnesota 17-25

Simmons J.S. (1926) A culture medium for differentiating organisms of the typhoid-colon Aerogenes groups and for isolation of certain fungi. $J$. Infect. Dis. 39, 209-214

Spiers A.G. (1979) Isolation and characterization of Agrobacterium species. N. Z. J. Agric Res. 22, 631636

White L.O. (1972) The taxonomy of the crown-gall organism Agrobacterium tumefaciens and its relationship to Rhizobia and other Agrobacteria. J. Gen. Microbiol. 72, 565-574 